\section{Transmission Electron Microscopy of Beryllium}

WE have prepared thin films of beryllium in which it is possible to observe directly the arrangements of dislocations and other structural defects by transmission electron microscopy. The films were made by two methods.

Beryllium was deposited as thin $(0.03-0.05 \mathrm{~mm}$.) polycrystalline flake on cool surfaces from metal melted by electron bombardment. This flake was etched in a dilute aqueous solution of hydrofluoric and nitric acids to give specimens suitable for examination. The grain-size was about $1 \mu$. Single-crystal specimens were prepared by cleaving a beryllium crystal parallel to the basal plane to obtain a flake which was ground and polished down to a thickness of $0.02 \mathrm{~mm}$. and then etched. Specimens were examined using $70-\mathrm{kV}$. electrons in the prototype Metropolitan-Vickers E.M.6 electron microscope.

Grain boundaries and single perfect dislocations were clearly visible in the evaporated flake. Many grains were structurally perfect while others contained several dislocations. From the electron-optical fringes on the grain boundaries the films were shown to be several thousand angstrom units thick.

A low-magnification electron micrograph of a singleerystal thin film is shown in Fig. 1. The long, sharp, roughly parallel lines make a [1]00] trace on the basal plane. From this and the fact that they make a line-projection on (0001), it may be concluded that they are either due to single dislocation lines or to [1150] planes. As the difference in contrast on either side of them is often quite marked (it is similar to that on crossing a well-formed sub-boundary in aluminium ${ }^{1,2}$ or germanium ${ }^{3}$ ) there must be a considerable misorientation associated with them. Furthermore, the contrest on them is higher than that usually associated with single dislocations ; they are therefore planes of a $(1120)$-type. X-ray and conventional metallographic techniques have revealed the existence of $\{1120\}$ planes which are effectively tilt boundaries, and which are believed to be important in the fracture mechanisms observed in beryllium ${ }^{4}$. At points such as $A$ in Fig. 1, a boundary with a $[1100]$ trace is observed to end in a fan-like array of dislocations. This can be understood if this boundary consisted of dislocations parallel to the basal plane. Coupling the X-ray and metallographic

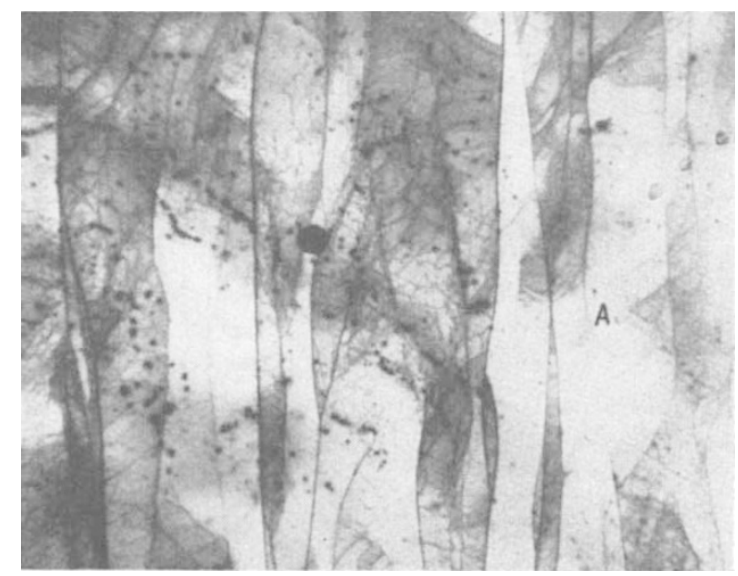

Fig. 1. Bend planes and dislocations in beryllium. At $\boldsymbol{A}$ the constituent dislocations of a bend plane fan out into an array. $(x 4,000)$

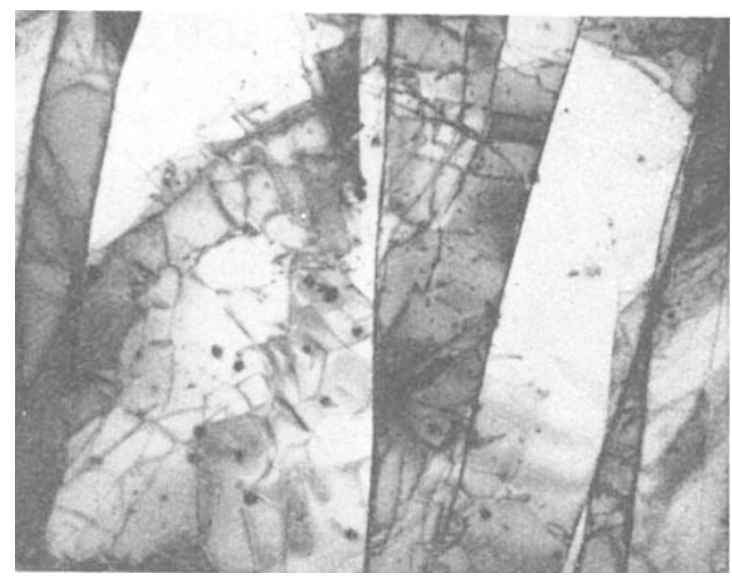

Fig. 2. Bend planes, dislocations and dislocation loops in beryllium. $(\times 40,000)$

evidence with the present observations one may conclude that the boundaries consist of edge dislocations stacked normal to the basal plane. It is yet to be determined whether these boundaries ('bend planes') are formed during the growth of the crystal from the melt or during cleavage.

Between the sharp parallel lines in Fig. 1 thore are randomly oriented lines believed, on the basis of similar studies of other materials, to be perfect dislocations more or less in the plane of the specimen. Fig. 2 shows bend planes and single dislocations at a higher magnification. Closed loops of dislocation line also occur ; these are probably due to the condensation of vacancies during solidification. The same mechanism probably accounts for the highly kinked nature of some of the dislocations.

The dislocation density in the single crystal was about $10^{8} / \mathrm{cm}^{2}$ : most of these dislocations were probably 'grown in'.
J. D. BAIRD
O. P. Hartree
R. Phitlips

Research Laboratory,

Associated Electrical Industries, Ltd.,

Aldermaston Court,

Aldermaston, Berkshire.

Oct. 7.

${ }^{3}$ Hirsch, P. B., Horne, R. W., and Whelan, H. J., Phil. Mag., 1, 67 (1956).

2 Bradley, D. E., and Phillips, R., Proc. Phys. Soc., B, 70, 533 (1957).

${ }^{3}$ Phillips, R. (to be published)

"Tuer, G. L., and Kaufmann, A. R., Chapter 7 in "The Metal Beryllim,", by white, I). W., and Burke, J. E. (A.S.M., 1953).

\section{Dispersion of the Stress Optical Coefficient of the Alkali Halides}

IN recent years the dispersion of the stress optical coefficients of diamond ${ }^{1}$, the alkali halides ${ }^{2}$ and magnesium oxide ${ }^{3}$ has been determined in the visible range of wave-lengths. The dispersion in fused quart $z^{4,5}$ has also been investigated from 5700 to $2500 \mathrm{~A}$. The observed dispersion is of the order of a few per cent for all these materials. In the present investigation the dispersion of the stress optical coefficient $C=n^{3}\left(q_{11}-q_{12}\right) / 2$ has been determ. ined from the visible range to $2400 \mathrm{~A}$. The method of measurement combines the differential feature of Bansigir and Iyengar's ${ }^{2}$ method with the photo. 